\title{
RILUZOLE PREVENTS ISCHEMIC SPINAL CORD INJURY CAUSED BY AORTIC CROSSCLAMPING
}

Loic Lang-Lazdunski, MD ${ }^{\mathrm{a}, \mathrm{b} *}$

Catherine Heurteaux, $\mathrm{PhD}^{\mathrm{b} *}$

Nathalie Vaillant, $\mathrm{BA}^{\mathrm{b}}$

Catherine Widmann, $\mathrm{BA}^{\mathrm{b}}$

Michel Lazdunski, PhD, DSc ${ }^{\mathrm{b}}$
Background: Recent studies support the involvement of glutamate neurotoxicity in the pathophysiology of spinal cord injury induced by aortic crossclamping. We investigated the effects of riluzole, a neuroprotective drug that blocks glutamatergic neurotransmission, in a rabbit model of spinal cord ischemia. Methods: The infrarenal aortas of New Zealand White albino rabbits $(n=40)$ were occluded for 40 minutes. Experimental groups were as follows: sham operation group $(n=5)$, control group undergoing occlusion but receiving no pharmacologic intervention $(n=10)$, experimental group $A(n=10)$ receiving $8 \mathrm{mg} / \mathrm{kg}$ riluzole intravenously 30 minutes before ischemia, experimental group $\mathrm{B}(\mathrm{n}=10)$ receiving $4 \mathrm{mg} / \mathrm{kg}$ riluzole intravenously 30 minutes before ischemia and at the onset of reperfusion, and experimental group $\mathrm{C}$ ( $\mathrm{n}$ = 10) receiving $8 \mathrm{mg} / \mathrm{kg}$ riluzole intravenously at the onset of reperfusion. Neurologic status was assessed at 6, 24, and 48 hours after the operation and then daily until the fifth day. All animals were killed at 24, 48, or 120 hours after the operation. Spinal cords were harvested for histopathologic studies, immunohistochemical studies for microtubuleassociated protein 2 , and search for morphologic features of apoptosis by the terminal deoxynucleotidyltransferase-mediated deoxyuridine triphosphate-biotin nick-end labeling staining method. Results: All animals in the control group became paraplegic. Except for 1 rabbit in group $\mathrm{C}$, all riluzole-treated animals had better neurologic function. Luxol fast blue and terminal deoxynucleotidyltransferase-mediated deoxyuridine triphosphate-biotin nick-end labeling staining methods demonstrated typical morphologic changes characteristic of necrosis and apoptosis in control animals. Riluzole prevented or attenuated ischemia-induced necrosis, apoptosis, and cytoskeletal proteolysis, depending on the dose and the timing of administration. Conclusion: Riluzole may have therapeutic utility during high-risk operations on the thoracoabdominal aorta. (J Thorac Cardiovasc Surg 1999;117:881-9)
$S^{n}$ pinal cord ischemia remains a devastating complication of thoracoabdominal aortic operations, with paraplegia occurring after as many of $31 \%$ of proce-

From the Department of Cardiovascular Surgery, ${ }^{\mathrm{a}}$ Paris, and the Institute of Molecular and Cellular Pharmacology, ${ }^{\mathrm{b}}$ Valbonne, France.

Supported by the Centre National de la Recherche Scientifique and the Fondation pour la Recherche Médicale.

Received for publication Sept 22, 1998; revisions requested Nov 13 , 1998; revisions received Dec 11, 1998; accepted for publication Dec 11, 1998.

Address for reprints: Michel Lazdunski, PhD, DSc, Institut de Pharmacologie Moléculaire et Cellulaire, CNRS UPR 411, 660 route des Lucioles, Sophia-Antipolis, 06560 Valbonne, France.

*These authors contributed equally to this work.

Copyright @ 1999 by Mosby, Inc.

$0022-5223 / 99 \$ 8.00+0 \quad \mathbf{1 2 / 1 / 9 6 5 3 1}$ dures. ${ }^{1}$ The cellular and molecular mechanisms that underlie hypoxic-ischemic injury to the spinal cord have not been totally elucidated, but recent studies suggest that the release of excitatory amino acids by ischemic cells into the extracellular space of the central nervous system may contribute substantially to neuronal death. ${ }^{2,3}$

Glutamate is thought to be the primary excitatory amino acid in the spinal cord, and it may destroy neuronal cells through its actions on $N$-methyl-D-aspartate (NMDA) and non-NMDA receptors by inducing massive sodium and calcium ion influxes into the cell, resulting in neuronal death.,5 Microdialysis studies have confirmed that an elevation in glutamate level is induced by spinal cord ischemia. ${ }^{3}$ Although the neuroprotective effects of NMDA receptor antagonists have 
been demonstrated both in vitro and in vivo, ${ }^{6,7}$ their pronounced side effects limit their clinical use. ${ }^{7,8}$

Riluzole (2-amino-6-trifluoromethoxy benzothiazole) is a neuroprotective drug that inhibits both sodium and calcium ion channels, ${ }^{9}, 10$ activates a new class of background potassium ion channels, ${ }^{11}$ and blocks glutamatergic neurotransmission in the central nervous system by noncompetitively blocking the NMDA receptor. ${ }^{12,13}$ Riluzole has demonstrated anti-ischemic properties in several models of focal and global cerebral ischemia ${ }^{13-15}$ and is currently in clinical use among patients with amyotrophic lateral sclerosis. ${ }^{16,17}$ It seems devoid of major side effects, suggesting that it might be of use in clinical situations involving spinal cord ischemia.

The aim of this study was to determine whether riluzole administered before or after aortic occlusion was capable of preserving the structural integrity of the spinal cord in a well-established model of severe ischemia. ${ }^{18}$ In this setting it appeared worthwhile to determine whether riluzole could prevent ischemiainduced apoptosis ${ }^{19}$ and degradation of microtubuleassociated protein 2 (MAP2), a cytoskeletal protein responsible for maintaining the structural integrity of the neuron and an early marker of cytoplasmic damage induced by spinal cord injury. ${ }^{20}$

\section{Materials and methods}

Animal care and surgical technique. We used female New Zealand White albino rabbits (Charles River Laboratories, Inc, Wilmington, Mass) weighing 3.5 to $4.5 \mathrm{~kg}$. Animal care and experiments complied with the "Guide for the Care and Use of Laboratory Animals" prepared by the Institute of Laboratory Animal Resources and published by the National Institutes of Health (NIH Publication No. 86-23, revised 1985). Animals were anesthetized by the intramuscular administration of $50 \mathrm{mg} / \mathrm{kg}$ ketamine and $10 \mathrm{mg} / \mathrm{kg}$ xylazine. Animals were allowed to breath spontaneously, and general anesthesia was maintained by inhalation of $1 \%$ halothane mixed with oxygen administered by face cone at a rate of $6 \mathrm{~L} / \mathrm{min}$. After placement of 24-gauge ear vein and artery catheters, a flank incision was made and the infrarenal aorta was exposed through a retroperitoneal approach. Intravenous heparin $(150 \mathrm{U} / \mathrm{kg})$ was given and spinal cord ischemia was induced by crossclamping of the aorta with surgical microclamps (Biover vessel clips; Arex, Palaiseau, France) for 40 minutes. Arterial blood pressure and heartbeat were continuously monitored throughout the procedure (Hewlett-Packard monitor model 78353B; Hewlett-Packard Company, Palo Alto, Calif). Percutaneous arterial oxygen saturation was continuously monitored. Body temperature was continuously monitored with a flexible probe inserted $3 \mathrm{~cm}$ into the rectum. Core temperature was registered from the onset of anesthesia to 1 hour after reperfusion and supported by a heating lamp throughout the procedure. The aortic cross- clamps were removed after 40 minutes and the flank was closed in 2 layers. The bladders of the paraplegic animals were emptied twice daily with the Credé maneuver. Animals in the sham operation group $(n=5)$ were killed 3 hours after exposure of the aorta without crossclamping of the vessel. Animals were randomly assigned to be killed at 24,48 , or 120 hours. All animals were killed with a lethal intraperitoneal injection of $200 \mathrm{mg} / \mathrm{kg}$ pentobarbital. Spinal cords were quickly removed for histopathologic and immunohistochemical examinations. The L4-L5 segment was fixed in Holland Bouin solution. The L6-L7 segment was frozen in isopentane on dry ice and stored at $-70^{\circ} \mathrm{C}$.

Drug treatment. Riluzole (Research Biochemicals International, distributed by Bioblock Scientific, Illkirsch, France) was first dissolved in $0.1 \mathrm{~N}$ hydrochloric acid and then diluted in $0.9 \%$ saline solution. It was injected intravenously in all groups of rabbits. Experimental group A animals $(\mathrm{n}=10)$ received $8 \mathrm{mg} / \mathrm{kg}$ riluzole 30 minutes before aortic occlusion. Experimental group B animals $(\mathrm{n}=10)$ received $4 \mathrm{mg} / \mathrm{kg}$ riluzole 30 minutes before aortic occlusion and at the onset of reperfusion. Experimental group $\mathrm{C}$ animals received $8 \mathrm{mg} / \mathrm{kg}$ riluzole at the onset of reperfusion. Control animals $(n=10)$ underwent standard aortic occlusion and intravenous injection of $0.9 \%$ sodium chloride $30 \mathrm{~min}$ utes before ischemia (volume and conditions identical to those of riluzole injection). A separate sham operation group $(\mathrm{n}=3)$ received $8 \mathrm{mg} / \mathrm{kg}$ riluzole to verify that the drug itself did not induce histologic changes in the spinal cord.

Evaluation of neurologic outcome. Neurologic status was scored by assessment of hind limb neurologic function according to the Tarlov scale ${ }^{21}(0$, no movement; 1 , slight movement; 2 , sits with assistance; 3 , sits alone; 4 , weak hop; 5 , normal hop) at 24,48 , or 120 hours after ischemia. Two observers (1 of whom was blinded to the experimental conditions) graded the neurologic status independently.

Statistical analysis. Statistical analyses of physiologic and hemodynamic parameters were performed by analysis of variance for repeated measures. All hemodynamic data are expressed as mean $\pm \mathrm{SD}$. Statistical analyses of the neurologic scores were done with the nonparametric Mann-Whitney $U$ test.

Tissue preparation. For paraffin sections, spinal cords (L4-L5 segments) were removed and immersed in Holland Bouin solution for 24 hours. Specimens were dehydrated in alcohol $95 \%$ for 30 minutes, followed by 4 changes of $100 \%$ alcohol for 1 hour each and 5 changes of toluene for 1 hour each under vacuum at $37^{\circ} \mathrm{C}$. Spinal cords were infiltrated with paraffin (4 changes of paraffin for 1 hour each) and embedded in paraffin at $57^{\circ} \mathrm{C}$ under vacuum and pressure. Sections were cut on a microtome (Leica Technology BV, Rijswijk, The Netherlands) at $7 \mu \mathrm{m}$.

For cryostat sections, spinal cords (L6-L7 segments) were quickly extracted and freshly frozen in isopentane at $-45^{\circ} \mathrm{C}$. Cryostat coronal sections $(10 \mu \mathrm{m})$ were mounted on poly-Llysine-coated slides and tissue was postfixed by successive immersions in $0.01 \mathrm{~mol} / \mathrm{L}$ phosphate-buffered saline solution (PBS) and 4\% paraformaldehyde for 30 minutes. Sections were then dehydrated in ethanol baths $(50 \%, 70 \%$, and $100 \%$ ), air dried, and stored at $-70^{\circ} \mathrm{C}$ until use. 
Table I. Neurologic status at 24 hours after ischemia

\begin{tabular}{lccccc}
\hline $\begin{array}{c}\text { Sharlov } \\
\text { score }\end{array}$ & $\begin{array}{c}\text { operation } \\
(n=5)\end{array}$ & $\begin{array}{c}\text { Control } \\
(n=10)\end{array}$ & $\begin{array}{c}A^{* \dagger} \\
(n=10)\end{array}$ & $\begin{array}{c}B^{*+} \\
(n=10)\end{array}$ & $\begin{array}{c}C \S \\
(n=10)\end{array}$ \\
\hline 0 & - & 10 & - & - & 1 \\
1 & - & - & - & 1 & 3 \\
2 & - & - & 3 & - & 1 \\
3 & - & - & 2 & - & - \\
4 & - & - & - & 1 & 4 \\
5 & 5 & - & 5 & 8 & 1 \\
\hline
\end{tabular}

Neurologic status was scored by assessment of hind limb neurologic functions according to the Tarlov scale ${ }^{21}$ ( 0 , no movement; 1 , slight movement; 2 , sits with assistance; 3, sits alone; 4, weak hop; 5, normal hop). Experimental groups were as follows: sham operation group with no aortic crossclamping, control group receiving no pharmacologic intervention, experimental group A $(\mathrm{n}=10)$ receiving $8 \mathrm{mg} / \mathrm{kg}$ riluzole intravenously 30 minutes before ischemia, experimental group B $(\mathrm{n}=10)$ receiving $4 \mathrm{mg} / \mathrm{kg}$ riluzole intravenously 30 minutes before ischemia and at the onset of reperfusion, experimental group $\mathrm{C}$ ( $\mathrm{n}=10$ ) receiving $8 \mathrm{mg} / \mathrm{kg}$ riluzole intravenously at the onset of reperfusion. $* P=.0001$ compared with control group by Mann-Whitney $U$ test.

$\dagger P=.2004$ compared with experimental group B by Mann-Whitney $U$ test. $\ddagger P=.0044$ compared with experimental group C by Mann-Whitney $U$ test. $\S P=.0003$ compared with control group by Mann-Whitney $U$ test.

All spinal cords were divided into 2 groups, 1 for frozen sections and 1 for paraffin sections. For each spinal cord studied 6 sections were placed on 3-aminopropylethoxysilane-coated slides and 10 slides per rabbit (randomly chosen) were used in each stage of the analysis. All samples processed for histopathologic and immunohistochemical experiments were taken from animals examined for functional recovery. The neuropathologist, who was blinded to experimental conditions, performed the histologic assessment by means of light microscopy.

Histopathologic examination. The Klüver-Barrera Luxol fast blue staining method ${ }^{22}$ was used. Transverse $7-\mu \mathrm{m}$ sections were deparaffinized by washing twice in xylene for 5 minutes each and heating at $56^{\circ} \mathrm{C}$ overnight and were then rehydrated through a graded series of alcohols and distilled water. Sections were soaked in $70 \%$ alcohol for 10 minutes. They were stained at $57^{\circ} \mathrm{C}$ for 16 hours in solution A, containing $1 \mathrm{mg}$ Luxol fast blue (Solvent Blue 38; Sigma Chemical Co, St Louis, Mo) and $5 \mathrm{~mL} \mathrm{10 \%} \mathrm{acetic} \mathrm{acid} \mathrm{in}$ $1000 \mathrm{~mL} \mathrm{95 \%} \mathrm{alcohol.} \mathrm{Sections} \mathrm{were} \mathrm{then} \mathrm{rinsed} \mathrm{in} \mathrm{95 \%}$ alcohol and distilled water. They were differentiated by being dipped singly into $0.05 \%$ aqueous lithium carbonate solution for a few seconds, then washed through several changes of $70 \%$ alcohol, and then placed in distilled water. Sections were then counterstained with neutral red stain (Sigma). Sections were treated with $0.2 \%$ sodium bisulfite for 1 minute and then immersed in $0.1 \mathrm{~mol} / \mathrm{L}$ acetate buffer $(\mathrm{pH}$ 5.6) for 1 minute. Neutral red staining was performed in solution B, containing 3 volumes of $0.05 \%$ neutral red stock solution and 2 volumes of $0.1 \mathrm{~mol} / \mathrm{L}$ acetate buffer, for $15 \mathrm{~min}$ utes at room temperature. Sections were rinsed in distilled water, immersed in copper sulfate-chrome alum solution for 1 second, and rinsed in distilled water. Sections were then mounted in Aquamount (Biomeda, Foster City, Calif).
Table II. Physiologic parameters

\begin{tabular}{lcccc}
\hline & $\begin{array}{c}\text { Control } \\
(n=10)\end{array}$ & $\begin{array}{c}A \\
(n=10)\end{array}$ & $\begin{array}{c}B \\
(n=10)\end{array}$ & $\begin{array}{c}C \\
(n=10)\end{array}$ \\
\hline $\begin{array}{l}\text { Weight }(\mathrm{kg}) \\
\begin{array}{l}\text { Rectal temperature* } \\
\left({ }^{\circ} \mathrm{C}\right)\end{array}\end{array}$ & $3.92 \pm 0.8$ & $3.89 \pm 0.9$ & $4 \pm 0.7$ & $3.94 \pm 0.8$ \\
$\quad$ Maximum & $39.2 \pm 0.5$ & $39.2 \pm 0.3$ & $39.3 \pm 0.2$ & $39.4 \pm 0.3$ \\
$\quad$ Minimum & $38.3 \pm 0.6$ & $38.3 \pm 0.3$ & $38.4 \pm 0.4$ & $38.3 \pm 0.2$ \\
$\begin{array}{l}\text { Heart rate } \\
\text { (beats/min) }\end{array}$ & & & & \\
$\begin{array}{l}\text { Maximum } \\
\text { Minimum }\end{array}$ & $192 \pm 7$ & $195 \pm 7$ & $191 \pm 12$ & $192 \pm 16$ \\
Mean arterial blood \\
pressure (mm Hg)
\end{tabular}

Values are expressed as mean \pm SD. Groups are as given in the footnote to Table I.

*Maximum and minimum rectal temperatures were taken from those recorded from the onset of anesthesia to 1 hour of reperfusion.

DNA nick-end labeling by terminal deoxynucleotidyltransferase-mediated deoxyuridine triphosphate-biotin nick-end labeling (TUNEL) reaction was also used. Coronal $10-\mu \mathrm{m}$ frozen sections were used and processed according to the TUNEL method. ${ }^{23}$ In brief, sections were rehydrated in ethanol $(95 \%, 70 \%$, and $50 \%)$ followed by PBS and bathed in $0.3 \%$ hydrogen peroxide in methanol to inactivate endogenous peroxidase. Sections were then rendered permeable in $0.3 \%$ polysorbate and PBS and washed twice in PBS before application of the TUNEL reaction mixture (in situ cell death detection kit, distributed by Hoffmann-La Roche Ltd, Basel, Switzerland). Positive control was obtained by advance incubation of a section with DNase I $(20 \mu \mathrm{g} / \mathrm{mL})$ for 15 minutes at room temperature before incubation with biotinylated deoxyuridine triphosphate. All slides were incubated in a humid chamber at $37^{\circ} \mathrm{C}$ for 2 hours. Sections were then washed twice in PBS and allowed to incubate overnight at $4^{\circ} \mathrm{C}$ with the secondary antifluorescein-peroxidase conjugate. On the following day sections were washed 3 times with PBS and the peroxidase labeling was revealed with 3-amino-9-ethyl carbazole by means of the VectaStain ABC kit (Vector Laboratories, Inc, Burlingame, Calif). After a final rinse in distilled water, sections were coverslipped.

Immunohistochemical examination for microtubuleassociated protein-2. Frozen sections $(25 \mu \mathrm{m})$ were immersed in $0.3 \%$ hydrogen peroxide and PBS for $10 \mathrm{~min}-$ utes, blocked with 5\% goat serum (Vector Laboratories, distributed by BIOSYS, Compiegne, France) and 3\% Triton for 1 hour at room temperature, and then rinsed in PBS 1X. Sections were then incubated with the primary antibody overnight. The antiserum used for the study of cytoskeletal protein expression (microtubule-associated protein-2 [MAP2]) was a monoclonal mouse anti-MAP2 (clone HM-2, diluted 1:500; Sigma). After the primary incubation and 3 rinses in PBS $1 \mathrm{X}$, sections were then incubated in biotinylated horse antimouse immunoglobulin G (diluted 1:100; Vector Laboratories) for 3 hours. MAP2 expression was visualized by $3^{\prime}$-diaminobenzidine and nickel chloride staining with the 

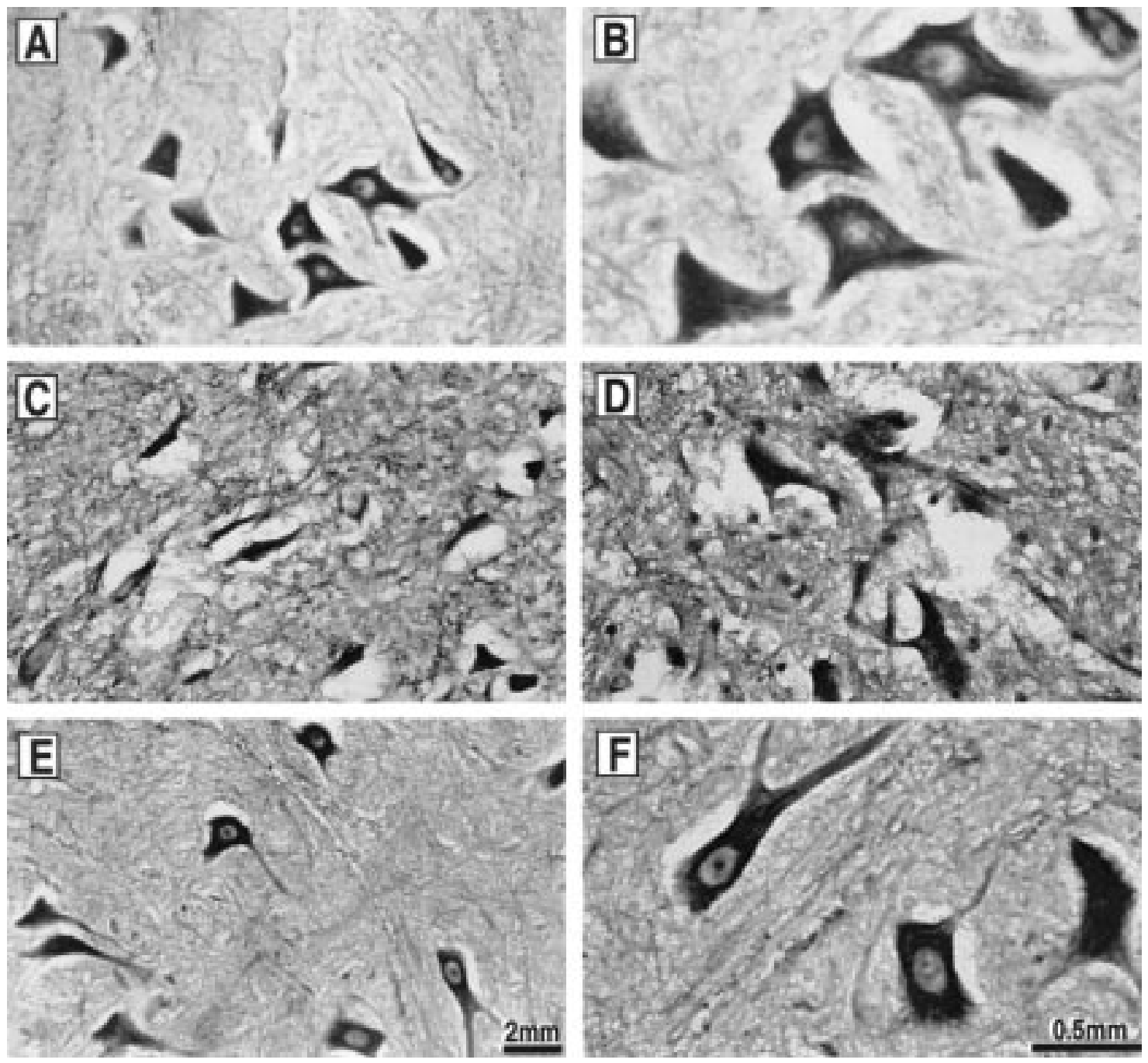

Fig 1. Representative photomicrographs of lumbar spinal cord sections stained with the Klüver-Barrera staining method from rabbits in sham operation ( $\mathbf{A}$ and $\mathbf{B}$ ), control (saline solution treatment, $\mathbf{C}$ and $\mathbf{D}$ ), and experimental riluzole treatment $(\mathbf{E}$ and $\mathbf{F})$ groups. All but sham operation group underwent 40 minutes of ischemia and 5 days of reperfusion. Chosen section for illustration of spinal cords in riluzole-treated rabbits is that of a rabbit with Tarlov score of 5 that was treated 30 minutes before aortic occlusion and at onset of reperfusion. $\mathbf{A}$ and $\mathbf{B}$, Normal appearance of motor neurons in ventral horn of rabbits in sham operation group. $\mathbf{C}$ and $\mathbf{D}$, Necrotic motor neurons in ventral horn of control rabbits. $\mathbf{E}$ and $\mathbf{F}$, Ventral horn motor neurons of riluzole-treated rabbits appear completely normal.

VectaStain ABC kit (Vector Laboratories). All sections were washed a final time, in PBS $1 \mathrm{X}$ and then in distilled water, and mounted with glycerol.

\section{Results}

Neurologic outcomes. The results are summarized in Table I. At 24 hours after 40 minutes of ischemia, all rabbits in the control group $(n=10)$ showed paralysis of the hind limbs (Tarlov score of 0 ). Riluzole treat- ment remarkably enhanced the recovery of motor function in the hind limbs. Except for 1 rabbit in group $\mathrm{C}$, all animals treated with riluzole demonstrated significantly better neurologic scores after reperfusion than did untreated (control) rabbits. The best result was obtained with animals that received $4 \mathrm{mg} / \mathrm{kg}$ riluzole 30 minutes before aortic occlusion and at the onset of reperfusion (group B). Eighty percent of group B animals showed intact neurologic function (Tarlov score 

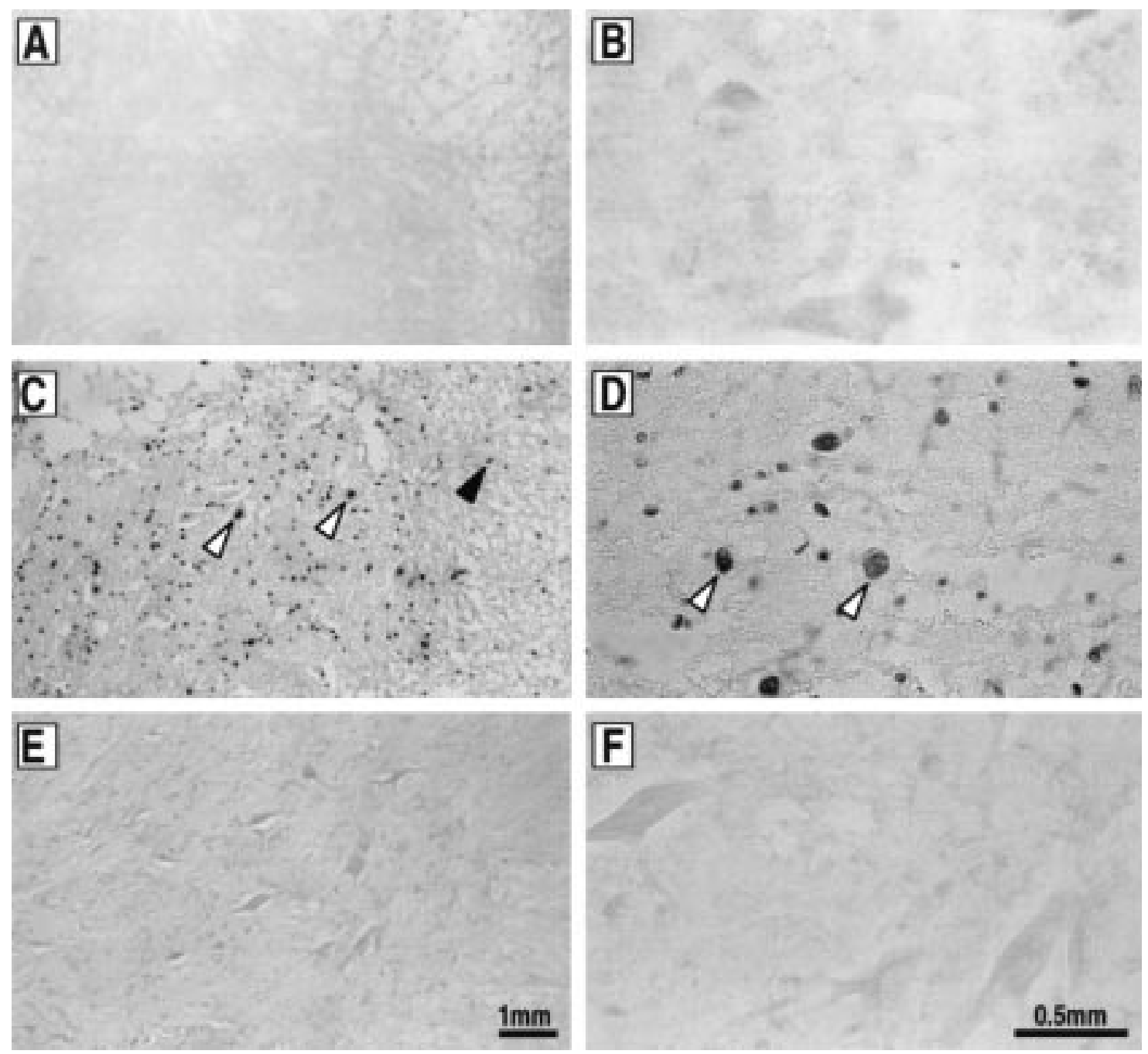

Fig 2. Representative photomicrographs show morphologic features of apoptosis with TUNEL staining in lumbar spinal cord sections from rabbits in sham operation (A and $\mathbf{B}$ ), control (saline solution treatment, $\mathbf{C}$ and $\mathbf{D}$ ), and experimental riluzole treatment $(\mathbf{E}$ and $\mathbf{F}$ ) groups. All but sham operation group underwent 40 minutes of ischemia and 5 days of reperfusion. Chosen section for illustration of spinal cords in riluzole-treated rabbits is that of a rabbit with Tarlov score of 5 that was treated 30 minutes before aortic occlusion and at onset of reperfusion. TUNEL-staining cells are characterized by a strong perinuclear graining pattern in gray (white arrow) and white (black arrow) matter of spinal cord. No TUNEL-staining cells could be detected in sham operation (A and B) or riluzole treatment (E and $\mathbf{F})$ groups. Control rabbit spinal cords exhibited numerous TUNEL-staining neurons and glial cells in both gray and white matter $(\mathbf{C}$ and $\mathbf{D})$.

of 5). This experimental group showed a significantly better recovery of neurologic function than that of experimental group $\mathrm{C}$, which was treated with $8 \mathrm{mg} / \mathrm{kg}$ riluzole after ischemia $(P<.01)$.

Physiologic and hemodynamic parameters. Physiologic parameters are presented in Table II. Rectal temperature, heart rate, and mean arterial blood pressure were not affected by the administration of riluzole.
There were no statistical differences in the physiologic parameters of the animals among the 4 experimental groups. Arterial oxygen saturation was within the reference range in all animals.

Histopathologic examination and terminal deoxynucleotidyltransferase-mediated deoxyuridine triphosphate-biotin nick-end labeling staining. Klüver-Barrera and TUNEL staining methods were 

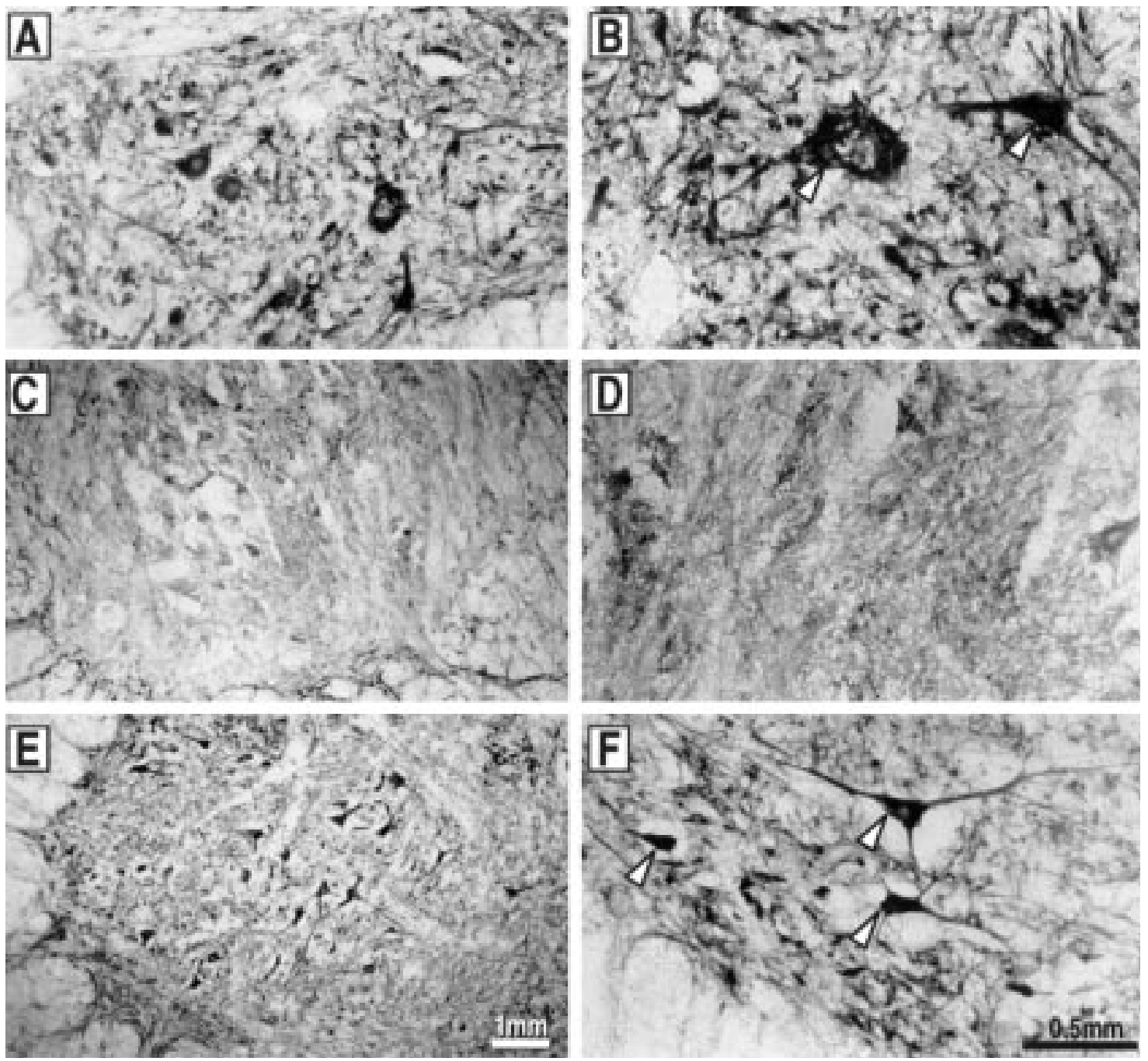

Fig 3. MAP2 immunoreactivity in lumbar spinal cord sections from rabbits in sham operation (A and $\mathbf{B})$, control (saline solution treatment, $\mathbf{C}$ and $\mathbf{D}$ ), and experimental riluzole treatment ( $\mathbf{E}$ and $\mathbf{F}$ ) groups. All but sham operation group underwent 40 minutes of ischemia and 5 days of reperfusion. Chosen section for illustration of spinal cords in riluzole-treated rabbits is that of a rabbit with Tarlov score of 5 that was treated 30 minutes before aortic occlusion and at onset of reperfusion. Intense MAP2 immunoreactivity was observed in dendrites and neuronal soma (arrow) of rabbits in sham operation (A and $\mathbf{B})$ and riluzole treatment $(\mathbf{C}$ and $\mathbf{D})$ groups. Control rabbit spinal cords ( $\mathbf{C}$ and $\mathbf{D})$ exhibited complete losses of MAP2 immunoreactivity in ventral and dorsal horns.

used to characterize necrosis and apoptosis, respectively, occurring in spinal cord injury after ischemia. Fig 1 shows representative Klüver-Barrera staining examples observed in the white and gray matter of the spinal cord in animals killed 5 days after the ischemic insult. No sign of neuronal damage was observed in the spinal cords of rabbits in the sham operation group (Fig 1, A and $B$ ). After 40 minutes of spinal cord ischemia, typical morphologic changes characteristic of necrosis appeared in paraplegic rabbits (Fig 1, $C$ and $D$ ). Injured spinal cord showed invasion of white blood cells into gray and white matter, cellular disruption and vacuolization of the gray matter, presence of shrunken necrotic neurons in ventral and dorsal horn gray matter, and infiltration by polymorphonuclear neutrophils and monocytes at 5 days after ischemia. Riluzole treatment almost completely protected spinal cord against the effects of ischemia. Fig 1, $E$ and $F$, shows that signs of necrosis were not present in the spinal cords of riluzole-treated animals (group B), which appeared histologically comparable to the spinal cords of rabbits in the sham operation group. 
Apoptosis characterized by chromatin condensation, DNA fragmentation, nuclear shrinkage, and fragmentation of nuclear bodies (apoptotic bodies) was visualized by the in situ TUNEL technique. Fig 2, $C$ and $D$, shows the presence of TUNEL-staining nuclei in gray and white matter of the spinal cord 48 hours after ischemia, whereas none of the nuclei in the control preparation showed TUNEL labeling (Fig 2, $A$ and $B$ ). Most TUNEL-staining neurons were located in dorsal horns and intermediate gray matter. No motor neurons were found to stain with TUNEL. Riluzole treatment significantly prevented ischemia-induced apoptosis in all sectors of the spinal cord. Fig 2, $E$ and $F$, demonstrates the lack of apoptotic nuclei in white and gray matter of the spinal cord at 48 hours of reperfusion in a riluzole-treated animal (group B). Most animals in groups A and B had no injury or minimal injury of the motor neurons, whereas $50 \%$ of the animals in group $\mathrm{C}$ had significant injury of the motor neurons (>50\% necrotic neurons). There were no differences in the locations of TUNELstaining neurons among animals in groups $\mathrm{A}, \mathrm{B}$, and $\mathrm{C}$. However, animals in group $\mathrm{C}$ had more TUNEL-staining cells in intermediate gray matter and dorsal horns than did animals in groups A and B.

Cytoskeletal proteolysis. The cytoskeletal element MAP2 located in neuronal soma and dendrites is involved in maintaining neuronal structural integrity, which is essential for normal cellular function and survival. Cytoskeletal degradation is highly sensitive to glutamate- and calcium ion-mediated excitotoxic events leading to activation of calcium ion-dependent phosphatases and proteases. ${ }^{20} \mathrm{Fig} 3, A$ and $B$, shows that the spinal cords of animals in the sham operation group exhibited intense perikaryal and dendritic MAP2 immunoreactivity. Aortic occlusion led to a rapid cytoskeletal protein degradation in control animals (Fig $3, C$ and D.) As early as 24 hours after spinal cord ischemia there was a loss of MAP2 antigenicity in cell bodies and dendrites of ischemic spinal cord neurons in control animals (Fig 3, $C$ and $D$ ). Riluzole treatment prevented or attenuated the cytoskeletal proteolysis in the ischemic spinal cords, depending on the timing of administration and dose. Fig 3, $E$ and $F$, shows that in spinal cord-injured rabbits treated with riluzole, MAP2 immunoreactivity was comparable to that in animals in the sham operation group. Administration of riluzole to a separate group of rabbits that underwent sham operation $(n=3)$ had no effect on MAP2 labeling (data not shown).

\section{Discussion}

Numerous surgical techniques and pharmacologic interventions have been used to reduce the rate of perioperative paraplegia after operations on the thoracoab- dominal aorta, but none can claim consistently proven efficacy. ${ }^{24}$ This report is the first to provide evidence that riluzole administered before or after aortic occlusion improves the neurologic status and preserves spinal cord integrity. The rabbit model of spinal cord ischemia used in this study is a reliable and reproducible model for producing neurologic deficits ${ }^{18}$ and testing drugs that might serve to protect the spinal cord from ischemic injury. Occlusion of the infrarenal aorta for 40 minutes leads to complete paraplegia, with Tarlov scores of 0 , in all control animals. To assess the impressive neuroprotective effect of riluzole, the motor function of the hind limbs was scored and the necrotic and apoptotic neuronal cell deaths associated with changes in the expression of MAP2 cytoskeletal protein induced by ischemia were analyzed in the spinal cords of both control and treated animals.

Evidence has accumulated that the excitatory amino acids, particularly glutamate, have potent neurotoxic activity during such conditions of depleted cellular energy as hypoxia and ischemia. ${ }^{4,5}$ The ischemia-induced overaccumulation of glutamate and other excitatory amino acids in the extracellular space of the central nervous system leads to excessive excitation of the NMDA and non-NMDA receptors, leading to a rise in intracellular calcium that triggers proteases, lipases, protein kinase $\mathrm{C}$, nitric oxide synthase, endonucleases, altered gene transcription, and release of free radicals, eventually producing neuronal injury and death. ${ }^{4}$ Much attention has recently focused on NMDA and non-NMDA receptor antagonists, which have been shown to be neuroprotective in several models of cerebral or spinal cord ischemia. ${ }^{2-6}$ Some of these compounds have major side effects, however, limiting their clinical use. ${ }^{7,8,25}$

Riluzole is a neuroprotective drug, although its exact molecular target has not been yet identified. An important and probably indirect effect of riluzole is the inhibition of presynaptic glutamate release. ${ }^{12,13}$ This inhibition, which leads to a neuroprotective effect, is probably linked to the capacity of riluzole to inhibit both sodium and calcium ion voltage-sensitive channels ${ }^{9,10}$ and to activate a new class of background potassium ion channels that are highly expressed in the spinal cord. ${ }^{11}$ At the postsynaptic level riluzole is probably effective on the same types of ion channels and would thus prevent some of the deleterious postsynaptic effects of glutamate by opening background potassium ion channels ${ }^{11}$ and producing postsynaptic hyperpolarization by increasing magnesium ion blockade of NMDA receptors. ${ }^{26}$ In addition, a brief report recently indicated that administration of riluzole after spinal cord injury improves mitochondrial function and increases sodium ion-dependent glutamate reuptake. ${ }^{27}$ 
In vivo, riluzole has demonstrated neuroprotective action in several models of neurodegenerative disease, such as Parkinson and Huntington diseases, ${ }^{28-30}$ cerebral ischemia, ${ }^{14,15,31}$ and traumatic spinal cord injury. ${ }^{20,32}$ It has also been reported that riluzole at the dose used in this work $(8 \mathrm{mg} / \mathrm{kg})$ attenuates both neurologic motor and cognitive dysfunction after experimental brain injury. ${ }^{33}$ This report demonstrates that riluzole treatment results in improved spinal cord protection in a setting of severe ischemia. The analysis of the neurologic status demonstrates that the tolerance of the spinal cord to ischemia was significantly improved by riluzole in the 3 experimental groups. The best protection was obtained when riluzole was administered before ischemia; however, riluzole afforded significant protection even when administered shortly after ischemia. An intravenous injection of riluzole 30 minutes before aortic occlusion and at the onset of reperfusion led to the best Tarlov score and prevented paraplegia in almost $100 \%$ of animals, despite a 40-minute ischemic insult. Histologic examination of the spinal cords revealed either no evidence or very little evidence of injury in riluzole-treated rabbits, whereas spinal cords from control animals had evidence of extensive spinal cord injury with central gray matter necrosis, vacuolization, Nissl substance dissolution, eosinophilic cytoplasm, and monocyte and macrophage infiltration. This study also provides evidence that 40 minutes of ischemia resulted in the degradation of cytoskeletal MAP2, which is known to be an early event after spinal cord injury, ${ }^{20}$ and that riluzole treatment totally prevented MAP2 breakdown. This observation suggests that the spinal cord really is protected against ischemia-induced necrosis.

In addition to necrosis, neuronal cell death induced by ischemia also occurred through apoptosis. Programmed cell death, or apoptosis, is defined morphologically and biochemically by cellular shrinkage, chromatin condensation, and internucleosomal fragmentation of DNA. ${ }^{19}$ Apoptosis is the mode of cellular death that occurs during the normal development of tissues, including the spinal cord, and it has recently been demonstrated to be an important mode of cellular death in the ischemic spinal cord. ${ }^{19}$ In this study apoptotic cell death was detected by the observation of positively stained nuclei with the in situ end-labeling procedure (TUNEL method). Abundant TUNEL-staining cells were observed in ischemic spinal cords 48 hours after ischemia; again, riluzole treatment prevented apoptotic cell damage. In addition to establishing that riluzole is an antiapoptotic drug, these results indirectly establish a role for glutamate in the initiation of apoptotic cell death in spinal cord neurons.
Riluzole has been reported to slightly decrease body temperature when injected intravenously. ${ }^{13}$ It is well demonstrated that even mild hypothermia can increase the duration of ischemia tolerated ${ }^{34}$ by modulating the release of excitatory amino acids and by reducing the spinal cord metabolic rate for oxygen. ${ }^{35}$ In this study the body temperature was monitored in all animals from the moment that they were anesthetized to the moment that they were returned to their cages. All rabbits had a mild decrease in body temperature, but there was no significant difference between control and riluzole-treated animals. Hypothermia therefore does not account for the protective effects of riluzole seen in this study.

We anticipate that riluzole might have important clinical implications. This drug may be administered either prophylactically to patients undergoing high-risk thoracoabdominal aortic operations or curatively to patients with a ruptured aortic aneurysm or an aortic dissection and an evolving neurologic deficit. In the latter situation, riluzole might improve the neurologic outcome of patients by blocking the excitotoxic cascade in the spinal cord and by preserving numerous viable neurons while patients are undergoing emergency operation for aortic replacement and spinal cord revascularization.

Riluzole does nothing by itself to reestablish perfusion to ischemic areas. In the case of a neurologic deficit caused by aortic dissection, this drug would simply buy time until reperfusion could be established. Riluzole (100-200 mg/day) was made available for amyotrophic lateral sclerosis therapy in 1995. The drug has a few side effects, such as asthenia, mild sedative effects, and, more rarely, liver dysfunction. ${ }^{25,36}$ Some of these side effects might be more frequent at the doses of riluzole used in this study $(8 \mathrm{mg} / \mathrm{kg})$, but they should not limit the clinical application of riluzole as an acute treatment in the prevention of spinal cord injury during thoracoabdominal aortic operations.

We thank Frank Aguila and Yvette Benhamou for technical assistance. We thank Hôpital FOCH for use of the Experimental Surgery Laboratory.

\section{REFERENCES}

1. Svensson LG, Crawford ES, Hess KR, Coselli JS, Safi HJ. Experience with 1509 patients undergoing thoracoabdominal aortic operations. J Vasc Surg 1993;17:350-70.

2. Mori A, Ueda T, Nakamichi T, Yasudo M, Aeba R, Odaguchi H, et al. Detrimental effects of exogenous glutamate on spinal cord neurons during brief ischemia in vivo. Ann Thorac Surg 1997; 63:1057-62.

3. Rokkas CK, Helfrich LR, Lobner DC, Choi DW, Kouchoukos NT. Dextrorphan inhibits the release of excitatory amino acids during spinal cord ischemia. Ann Thorac Surg 1994;58:312-20.

4. Lipton SA, Rosenberg PA. Excitatory amino acids as a final com- 
mon pathway for neurologic disorders. N Engl J Med 1994;330: 613-22.

5. Regan RF, Choi DW. Glutamate neurotoxicity in spinal cord cell culture. Neuroscience 1991;43:585-91.

6. Kochhar A, Zivin JA, Lyden PD, Mazarella V. Glutamate antagonist therapy reduces neurologic deficits produced by focal central nervous system ischemia. Arch Neurol 1988;45:148-53.

7. Koroshetz WJ, Moskowitz MA. Emerging treatments for stroke in humans. Trends Pharmacol Sci 1996;17:227-33.

8. Olney JW, Labruyere J, Price MT. Pathological changes induced in cerebrocortical neurons by phencyclidine and related drugs. Science 1989;244:1360-2.

9. Benoit E, Escande D. Riluzole specifically blocks inactivated Na channels in myelinated nerve fibre. Pflugers Arch 1991;419:6039.

10. Huang CS, Song JH, Nagata K, Yeh JZ, Narahashi T. Effects of the neuroprotective agent riluzole on the high voltage-activated calcium channels of rat dorsal root ganglion neurons. J Pharmacol Exp Ther 1997;282:1280-90.

11. Fink M, Lesage F, Duprat F, Heurteaux C, Reyes R, Fosset M, et al. A neuronal two $\mathrm{P}$ domain $\mathrm{K}^{+}$channel stimulated by arachidonic acid and polyunsaturated fatty acids. EMBO J 1998;17: 3297-308.

12. Martin D, Thompson MA, Nadler JV. The neuroprotective agent riluzole inhibits release of glutamate and aspartate from slices of hippocampal area. Eur J Pharmacol 1993;250:473-6.

13. Doble A. The pharmacology and mechanism of action of riluzole. Neurology 1996;47:S233-41.

14. Pratt J, Rataud J, Bardot F, Roux M, Blanchard JC, Laduron PM, et al. Neuroprotective actions of Riluzole in rodent models of global and focal cerebral ischemia. Neurosci Lett 1992;140:225-30.

15. Wahl F, Allix M, Plotkine M, Boulu RG. Effect of riluzole on focal cerebral ischemia in rats. Eur J Pharmacol 1993;230:209-14.

16. Lacomblez L, Bensimon G, Leigh PN, Guillet P, Meininger V. For the ALS/Riluzole study group II. Dose-ranging study of riluzole in amyotrophic lateral sclerosis. Lancet 1996;347:1425-31.

17. Wokke J. Riluzole. Lancet 1996;348:795-809.

18. Zivin JA, DeGirolami U. Spinal cord infarction: a highly reproducible stroke model. Stroke 1980;11:200-2.

19. Mackey ME, Wu Y, Hu R, DeMaro JA, Jacquin MF, Kanellopoulos GK, et al. Cell death suggestive of apoptosis after spinal cord ischemia in rabbits. Stroke 1997;28:2012-7.

20. Springer JE, Azbill RD, Kennedy SE, George J, Geddes JW. Rapid calpain I activation and cytoskeletal protein degradation following traumatic spinal cord injury: attenuation with riluzole pretreatment. J Neurochem 1997;69:1592-600.

21. Tarlov IM. Acute spinal cord compression paralysis. J Neurosurg 1972;36:10-20

22. Klüver H, Barrera E. A method for the combined staining of cells and fibers in the nervous system. J Neuropathol Exp Neurol 1953;12:400-3.

23. Gavrieli Y, Sherman Y, Ben-Sasson SA. Identification of programmed cell death in situ via specific labeling of nuclear DNA fragmentation. J Cell Biol 1992;11:493-501.

24. Gharagozloo F, Larson J, Dausmann MJ, Neville RF Jr, Gomes MN. Spinal cord protection during surgical procedures on the descending thoracic and thoracoabdominal aorta: review of current techniques. Chest 1996;109:799-809.

25. Morris RG, Anderson E, Lynch GS, Baudry M. Selective impairment of learning and blockade of long term potentiation by $\mathrm{N}$ methyl-D-aspartate receptor antagonist, AP5. Nature 1986;319: 774-6.

26. MacDonald JF, Nowak LM. Mechanisms of blockade of excitatory amino acid receptor channels. Trends Pharmacol Sci 1990; 11:167-72.

27. Mu X, Azbill RD, Springer JE. Riluzole treatment following spinal cord injury improves mitochondrial function and increases glutamate and glucose uptake [abstract]. Soc Neurosci 1997;23: 1384.

28. Benazzouz A, Boraud T, Dubédat P, Boireau A, Stutzmann JM, Gross C. Riluzole prevents MPTP-induced parkinsonism in the rhesus monkey: a pilot study. Eur J Pharmacol 1995;284:299307.

29. Barnéoud P, Mazadier M, Miquet JM, Parmentier S, Dubédat P, Doble A, et al. Neuroprotective effects of riluzole on a model of Parkinson's disease in the rat. Neuroscience 1996;74:971-83.

30. Mary V, Wahl F, Stutzmann JM. Effect of riluzole on quinolinateinduced neuronal damage in rats: comparison with blockers of glutamatergic neurotransmission. Neurosci Lett 1995;1:92-6.

31. Malgouris C, Bardot F, Daniel M, Pellis F, Rataud J, Uzan A, et al. Riluzole, a novel antiglutamate, prevents memory loss and hippocampal neuronal damage in ischemic gerbils. J Neurosci 1989;9:3720-7.

32. Stutzmann JM, Pratt J, Boraud T, Gross C. The effect of riluzole on post traumatic spinal cord injury in the rat. Neuroreport 1996; 7:387-92.

33. McIntosh TK, Smith DH, Voddi M, Perri BR, Stutzmann J M. Riluzole, a novel neuroprotective agent, attenuates both neurologic motor and cognitive dysfunction following experimental brain injury in the rat. J Neurotrauma 1996;13:767-80.

34. Vacanti FX, Ames A. Mild hypothermia and $\mathrm{Mg}^{++}$protect against irreversible damage during CNS ischemia. Stroke 1984;15:695-8.

35. Matsumoto M, Iida Y, Sakabe T, Sano T, Ishikawa T, Nakakimura $\mathrm{K}$. Mild and moderate hypothermia provide better protection than a burst-suppression dose of thiopental against ischemic spinal cord injury in rabbits. Anesthesiology 1997;86:1120-7.

36. Castells LI, Gámez J, Cervera C, Guardia J. Icteric toxic hepatitis associated with riluzole [letter]. Lancet 1998;351:648. 